OPEN ACCESS

Edited by: Luigi Aloj,

University of Cambridge,

United Kingdom

Reviewed by:

Guolin Ma,

China-Japan Friendship Hospital,

China

Natale Quartuccio, ARNAS Ospedali Civico Di Cristina

Benfratelli, Italy

*Correspondence:

$\mathrm{Nan} \mathrm{Li}$

rainbow6283@sina.com

Zhi Yang

pekyz@163.com

${ }^{t}$ These authors have contributed equally to this work

Specialty section: This article was submitted to Cancer Imaging and Image-directed Interventions,

a section of the journal

Frontiers in Oncology

Received: 27 May 2021 Accepted: 20 October 2021 Published: 09 November 2021

Citation:

Zhou N, Guo X, Sun H, Yu B, Zhu H, Li N and Yang $Z$ (2021) The Value of

${ }^{18}$ F-FDG PET/CT and Abdominal PET/

MRI as a One-Stop Protocol in Patients With Potentially Resectable

Colorectal Liver Metastases.

Front. Oncol. 11:714948. doi: 10.3389/fonc.2021.714948

\section{The Value of ${ }^{18}$ F-FDG PET/CT and Abdominal PET/MRI as a One-Stop Protocol in Patients With Potentially Resectable Colorectal Liver Metastases}

\author{
Nina Zhou ${ }^{1+}$, Xiaoyi Guo ${ }^{1+}$, Hongwei Sun ${ }^{2}$, Boqi Yu ${ }^{1}$, Hua Zhu ${ }^{1}$, Nan $\mathrm{Li}^{1 *}$ and $Z$ Zhi Yang ${ }^{1 *}$ \\ ${ }^{1}$ Key Laboratory of Carcinogenesis and Translational Research (Ministry of Education/Beijing), NMPA Key Laboratory for \\ Research and Evaluation of Radiopharmaceuticals (National Medical Products Administration), Department of Nuclear \\ Medicine, Peking University Cancer Hospital and Institute, Beijing, China, ${ }^{2}$ United Imaging Research Institute of Intelligent \\ Imaging, Beijing, China
}

Purpose: The aim of this study was to evaluate the clinical value of simultaneous positron emission tomography/computed tomography (PET/CT) and abdominal positron emission tomography/magnet resonance imaging (PET/MRI) in the detection of liver metastases and extrahepatic disease (EHD) in patients with potentially resectable colorectal liver metastases (CLM).

Methods: Fifty-six patients with CLM underwent conventional imaging (chest and abdomen CT, liver contrast-enhanced CT or MRI) and PET imaging [fluorine-18 fluorodeoxyglucose $\left({ }^{18} \mathrm{~F}-\mathrm{FDG}\right) \mathrm{PET} / \mathrm{CT}$ and subsequent liver PET/MRI] for staging or restaging. Diagnostic ability of PET imaging was compared with conventional imaging. Abnormal findings were correlated with follow-up imaging and/or histology. The influence of the PET imaging findings was categorized for each patient in relation to operability and other significant findings. The clinical management included three modalities (surgery for resectable CLM, unresectable CLM with conversion treatment, and systemic therapy). The clinical impact of the imaging modality was analyzed. The operative histopathological analysis and/or imaging follow-up were performed as the standard of reference.

Results: This study enrolled a total of 56 patients (median age 60 years, $62.5 \%$ were male, 36 with colon cancer and 20 with rectal cancer). For EHD detection, PET/CT detected more EHD than conventional imaging (60.7\% vs. 46.4\%). PET/CT had different findings in 19 (33.9\%) patients, including downstaging in 4 (7.1\%) patients and upstaging in 15 (26.8\%) patients. For liver lesion detection, PET/MRI showed comparable detection ability with CE-MRI and CE-CT (99.5\%, 99.4\%, and 86.5\%, respectively) based on lesion analysis, much higher than PET/CT (47.5\%). PET imaging had a major impact in 10/56 (17.9\%) patients (4 from unresectable to resectable, 6 from resectable to unresectable) and a minor impact in 4/56 (7.1\%) patients for changing the surgery extent. The therapeutic strategies had been altered in a total of $14 / 56$ patients (25\%) after PET/CT and PET/MRI scans. 


\begin{abstract}
Conclusion: The results of this study indicate that simultaneous ${ }^{18} \mathrm{~F}-\mathrm{FDG}$ PET/CT and abdominal PET/MRI scans can provide accurate information regarding CLM status and $\mathrm{EHD}$, and can affect the management of $25 \%$ of the patients by changing the therapeutic strategies determined by conventional imaging. This new modality may serve as a new one-stop method in patients with potentially resectable CLM.
\end{abstract}

Keywords: colorectal cancer, liver metastasis, PET/CT, PET/MRI, clinical impact analysis

\section{INTRODUCTION}

Colorectal cancer (CRC) is one of the most common cancers. Long-term patient outcome is heavily influenced by the initial stage. Approximately $25 \%-30 \%$ of patients have hepatic metastases at presentation. Recurrent disease is seen in up to $30 \%$ of patients within 2 years of initial resection, in which the majority manifests as liver metastases (1). Liver resection for colorectal liver metastasis (CLM) is an established treatment and offers a realistic chance of disease-free survival. Five-year survival rates following liver resection have been reported at approximately $30 \%$ with a perioperative mortality rate of $<3 \%$ (2-4). Therefore, identifying the group of patients who would benefit from a liver resection for CLM is paramount. In addition, approximately $20 \%-30 \%$ of newly diagnosed patients with CRC present with synchronous metastases. Some extrahepatic disease no longer represents an absolute contraindication to surgery, but necessitates a wider operative field or second operation, if technically feasible, allowing a truly curative surgical resection (5-8).

Although practice varies between treatment centers, many lines of evidence suggest that the best method for detection of liver metastases from CRC are computed tomography (CT) and magnetic resonance imaging (MRI) (9). For lesions with a diameter of less than $10 \mathrm{~mm}$, MRI is a more sensitive modality than CT $(10,11)$, and specifically in hepatobiliary MRI with specific contrast enhancers (such as gadoxetate), showing a higher accuracy of lesion detection (12-15). Many studies have investigated the optimal modality for imaging hepatic metastases, finding pooled sensitivity on a per-lesion basis of $88 \%$ for MRI, $74 \%$ for CT, and $79 \%$ for positron emission tomography/computed tomography (PET/CT) $(9,16-18)$.

For the detection of extrahepatic metastases (EHD) and local recurrence at the site of the initial colorectal surgery, CT and PET/CT scans are used (19). A prospective randomized trial evaluating high-quality CT and PET imaging involving 263 patients showed only a $7.6 \%$ change in management following $\operatorname{PET}(20,21)$, while a retrospective analysis reported, in one-third (33.3\%) of patients, a change in intended curative therapy to palliative therapy, or vice versa (22).

The soft-tissue contrast provided by CT in a PET/CT scan may not be sufficient enough for small lesion detection in the clinical practice. Hence, PET and MRI hybrid units [positron emission tomography/magnet resonance imaging (PET/MRI)] were initially manufactured in 2011 and were soon approved in both the United States and the European Union. Until now, the evidence on the potential value and role of PET/MRI in clinical medicine is still accumulating (23-25). Evaluation of small liver lesions can be challenging using PET/CT due to low image contrast. As a potential remedy, PET/MRI may be used to detect small liver lesions with its high soft-tissue contrast and functional diffusion-weighted imaging sequence $(23,26,27)$.

The aim of this study was to evaluate the influence of ${ }^{18} \mathrm{~F}$ FDG PET/CT in the detection of EHD and diagnostic efficiency of PET/MRI in the detection of intrahepatic lesions in patients with potentially resectable CLM.

\section{MATERIALS AND METHODS}

\section{Patient Enrollment}

This study was performed under a single-center prospective imaging protocol and was approved by the Medical Ethics Committee of Peking University Cancer Hospital (ethical approval no. 2018KT110-GZ01). Between October 2019 and December 2020, patients referred to the hepatobiliary multidisciplinary team for consideration of liver resection for CLM after initial imaging (including CT of chest and either contrast-enhanced CT or MRI of abdomen) were enrolled. These candidates took an ${ }^{18}$ F-FDG PET/CT scan first, followed by a delayed abdomen PET/MRI scan. All patients were provided with written informed consent before study participation. Inclusion criteria for the study participation include any of the following conditions (Figure 1):

(a) ${ }^{18}$ F-FDG PET/CT for the staging or restaging of patients with colorectal cancer.

(b) Patients have undergone abdomen contrast-enhanced (CE) CT or MRI for liver metastasis detection, and the interval time between PET and CT/MRI was less than 30 days.

(c) If there were positive or indeterminate findings on PET/CT, these must have confirmatory evidence with either histology or follow-up imaging at least 3 months.

Additionally, patients with any of the following conditions have been excluded:

(a) Age $<18$ years or $>80$ years because of the ethical restriction;

(b) Contraindication to PET/MRI imaging;

(c) Insufficient imaging follow-up to confirm the metastasis lesion, or insufficient follow-up for clinical therapy. 


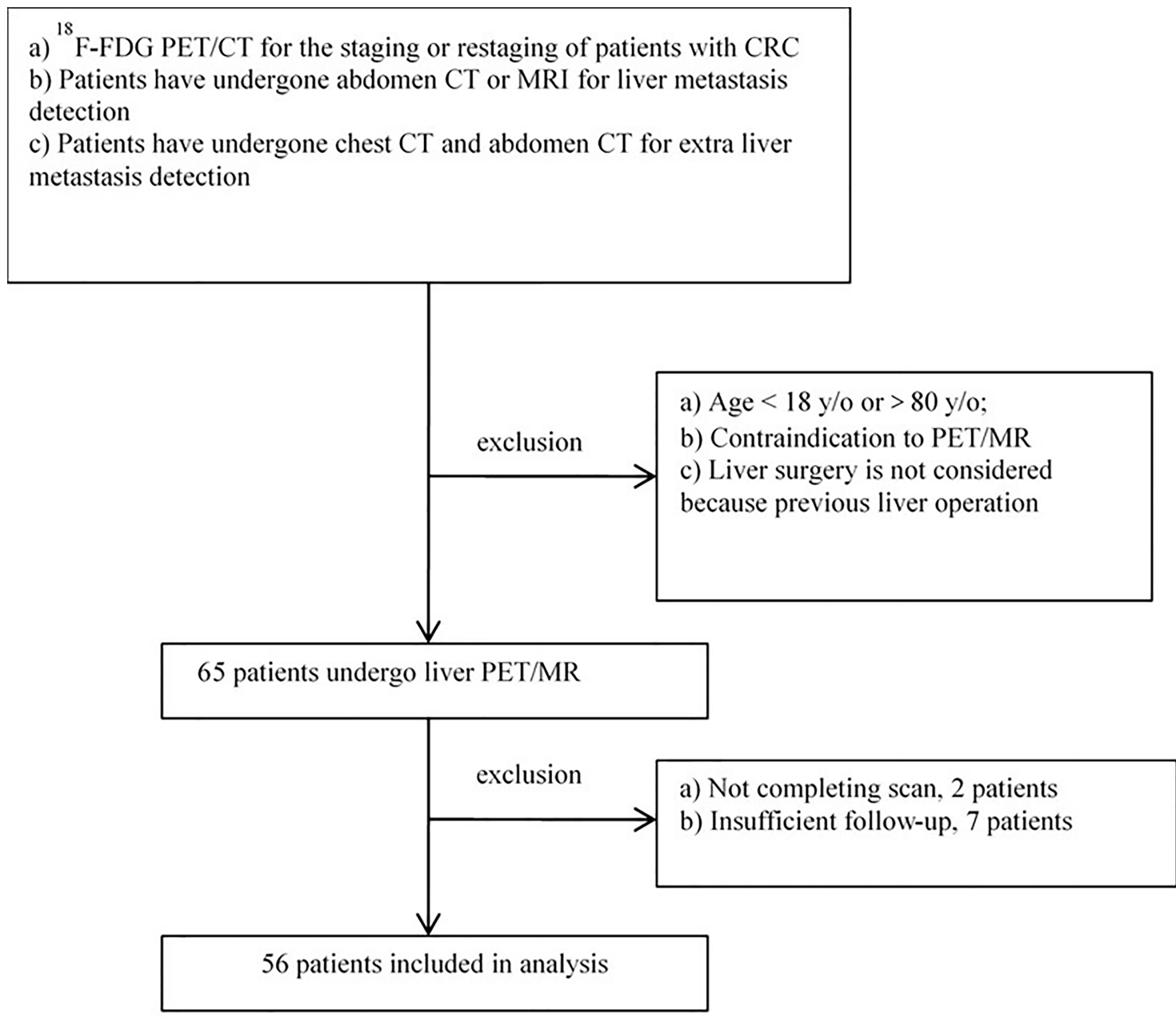

FIGURE 1 | The schematic of enrollment and exclusion criteria.

\section{Imaging}

Imaging was performed using a PET/CT scanner (Biograph64, SIEMENS, Erlangen, Germany) operated in 3D Flow Motion (bed entry speed $1 \mathrm{~mm} / \mathrm{s}$ ) from the apex of the skull to the midthigh, with a PET axial field of view of $21.6 \mathrm{~cm}$. The PET images were reconstructed by the TrueX + TOF method offered by the vendor. The CT component was a 64 slice spiral CT, and lowdose CT scans without contrast enhancement were acquired in CARE Dose 4D mode ( $120 \mathrm{kV}, 3.0 \mathrm{~mm}$ slice thickness). The patients were instructed to fast for at least $6 \mathrm{~h}$ before ${ }^{18} \mathrm{~F}-\mathrm{FDG}$ injection. In all cases, the serum glucose concentration met our institutional requirement $(\leq 140 \mathrm{mg} / \mathrm{dl})$. The injected activity was $3.7 \mathrm{MBq} / \mathrm{kg}$, and the time from injection to scan was $60 \mathrm{~min}$. The $\mathrm{PET} / \mathrm{CT}$ scan lasted about $15 \mathrm{~min}$.

${ }^{18} \mathrm{~F}$-FDG PET/MRI was performed on an integrated 3.0-T Timeof-flight PET/MRI scanner (uPMRI790, UIH, Shanghai, China). The scan started at $120-180 \mathrm{~min}$ after ${ }^{18} \mathrm{~F}-\mathrm{FDG}$ administration. The time interval between ${ }^{18} \mathrm{~F}$-FDG PET/CT scan and PET/MRI scan were $60-90 \mathrm{~min}$ in order to get a delay liver PET. Each patient underwent the same protocol as described in the following. Body array coil was placed around the individual and covered the entire liver. Respiratory gating was used in MRI acquisition whenever possible. PET reconstruction was conducted using a 3D-Ordered Subsets Expectation Maximization (3D-OSEM) algorithm applied on a $256 \times 256$ matrix. A four-compartment-model attenuation map ( $\mu$-map) automatically generated based on a water-fat-imaging MRI sequence was used for PET attenuation correction. The PET images were smoothed by a Gaussian filter with $3 \mathrm{~mm}$ full width at half maximum (FWHM). The MRI sequences were performed simultaneously with PET acquisition, including T2WI with fat saturation, T1WI, and DWI. The mean scan time for PET/MRI was $20 \pm 6 \mathrm{~min}$. The detailed MRI parameters are shown in Supplementary Table 1.

\section{Image Analysis}

Images were reviewed using our local picture archiving and communication system (PACS), by two accredited radiologists with more than 4 years of experience in hybrid PET/CT and PET/MRI imaging. In PET/CT imaging, lesions were rated as metastases when PET had positive uptake foci with abnormal 
density on CT. In PET/MRI imaging, liver lesions were rated as metastases when at least two of the three following criteria were met: (a) hyperintense on T2WI, (b) diffusion restriction on DWI, and (c) PET-positive. Moreover, CE-CT and CE-MRI images from PACS were reviewed by two radiologists. All liver lesions detected on PET/CT and PET/MRI were documented for patients with less than 10 lesions. Each patient was categorized into negative or positive of extrahepatic disease on PET/CT. The number/extent of liver metastasis on PET/MRI was also compared with CE-CT or CE-MRI scans.

\section{Analysis of Clinical Impact}

The conventional imaging (chest/abdomen CT and liver MRI/CT) and PET imaging (PET/CT and liver PET/MRI) were assessed by a multidisciplinary team (MDT), and then the patients were organized into three groups including (a) resectable CLM, (b) unresectable CLM with "conversion" as a strategic treatment goal, and (c) unresectable CLM with systematic therapy. They were further categorized as to whether the PET imaging findings had a major impact (change resectable to unresectable, change unresectable to resectable), minor impact (change resectable extent), or no impact (no therapy changed). For the patients who received liver surgery, the lesion number identified on imaging was compared with the final resection lesion number. The clinical treatment, biopsy, surgical pathologic analysis, correlation with prior imaging findings, and clinical and imaging follow-up were used as the reference standard for the image findings. The follow-up was conducted at least 90 days after the initial PET/CT and PET/ MRI study (Figure 2).

\section{RESULTS}

\section{Patient Characteristics}

Out of the 65 patients initially enrolled, 2 were excluded for an incomplete delayed PET/MRI scan, and another 7 were excluded for insufficient follow-up. Thus, 56 patients with colorectal liver metastases were included in the final analysis. Their median age was 59 years (31-80 years), where 36 patients had colon cancer and 20 patients had rectal cancer, with 21 patients receiving chemotherapy within 3 months. According to the reference standard and follow-up, a total of 206 liver metastatic lesions were detected, including 162 lesions with diameter less than or equal to $10 \mathrm{~mm}$ (range $2-10$, mean $6.5 \pm 1.9$ ), and 44 lesions with diameter larger than $10 \mathrm{~mm}$ (range 11-70, mean $19.9 \pm 12.8$ ). Clinical follow-up showed that 26 patients received surgery, 4 patients receives surgery after conversion treatment, and 26 patients received systematic therapy. The patient characteristics of this study are summarized in Table $\mathbf{1}$.

\section{PET/CT Imaging for Extrahepatic Disease Detection}

PET/CT detected EHD in 34 patients, 14 of which have oligometastatic disease (OMD). The conventional CT detected EHD in 26 patients.

Based on patient analysis, PET/CT had different findings in 19 patients, including downstaging in 4 patients and upstaging in 15 patients.

Based on lesion analysis, PET/CT detected more EHD for hilar lymph nodes (hilar LN) (4 vs. 3), lung metastases (lung M) (12 vs. 7), retroperitoneal lymph nodes (retroperitoneal LNs) (12 vs. 8), chest lymph nodes (chest LNs) (5 vs. 2), peritoneal nodules (13 vs. 6), and bone metastases (bone M) (2 vs. 0). PET/CT detected the same iliac lymph nodes, inguinal lymph nodes, and locoregional recurrent. PET/CT ruled out false-positive EHD, including one recurrence, one peritoneal nodule, one mediastinal lymph node, and one bone metastasis (Figure 3).

\section{Diagnostic Efficiency of PET/MRI for Liver Lesions}

CE-MRI was performed in 43 patients and CE-CT was performed in 13 patients for evaluation of liver lesion.

Based on patient analysis, PET/CT showed no metastasis in 16 out of 56 metastatic patients, while the other three image modalities (PET/MRI, CE-CT, and CE-MRI) detected liver lesions in all 56 patients. For one patient, CE-CT misdiagnosed the liver lesion as metastasis while PET/MRI showed a mass with restriction on DWI and low ${ }^{18} \mathrm{~F}-\mathrm{FDG}$ uptake on PET/CT. Then, a second primary tumor was diagnosed for the inconsistency with the high ${ }^{18}$ F-FDG uptake of primary lesion, which also

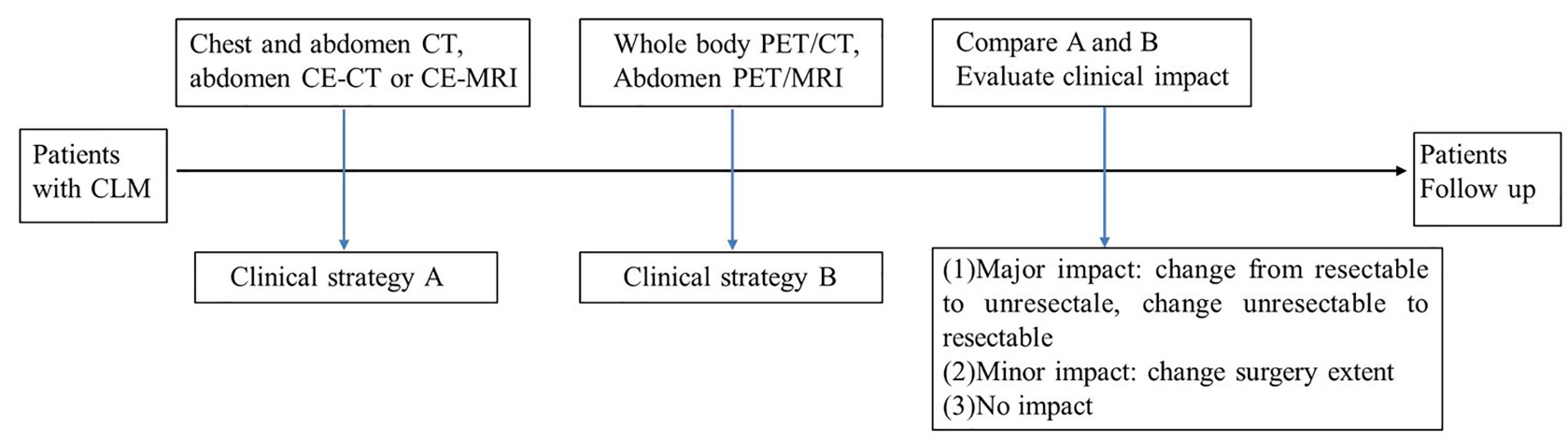

FIGURE 2 | The analysis flow chart of clinical impact. 
TABLE 1 | Patient characteristics of this study.

\begin{tabular}{lc}
\hline Patients Characteristic & Number \\
\hline Age (years) & $31-80$ (median 60) \\
Male/Female & $35 / 21$ \\
Primary tumor & 36 \\
Colon cancer & 20 \\
Rectal cancer & \\
Pathology subtypes & 50 \\
Moderate differentiated adenocarcinoma & 4 \\
Poorly differentiated adenocarcinoma & 2 \\
Mucinous adenocarcinoma & $7 / 49$ \\
Staging/Restaging & $21 / 35$ \\
Chemotherapy within 3 months (Yes/No) & \\
Conventional Imaging for CLM & 12 \\
CE-CT & 44 \\
CE-MRl & \\
Clinical follow-up & 26 \\
Surgery & 4 \\
Surgery after conversion treatment & 26 \\
Systematic therapy & \\
\hline
\end{tabular}

confirmed a second primary hepatic cell carcinoma (HCC) by pathology. PET/CT missed one or more lesions in 33 patients, regardless of whether the patients received therapy within 3 months or not $\left(\chi^{2}=2.17, p=0.141\right)$.

Figure 4 shows a patient with liver metastases detected by PET/MRI but missed on PET/CT. The PET/CT detected one lung lesion, so the patient received a simultaneous operation of lung and liver metastases.

For lesion analysis, there were 206 liver metastases based on standard reference. PET/MRI detected 205/206 (99.5\%) metastases, CE-MRI detected 168/169 (99.4\%), CE-CT detected 32/37 (86.5\%), and PET/CT only detected 98/206 (47.5\%). Both CE-MRI and PET/MRI detected three patients with bile duct infiltration (Figure 5) and two patients with multiple liver metastases that were unresectable (Table 2).

\section{The Clinical Impact of PET Imaging}

Based on conventional imaging, patients were organized into three groups including (a) resectable CLM $(n=32)$, (b) unresectable CLM with "conversion" as a strategic treatment goal $(n=4)$, and (c) unresectable CLM with systematic therapy $(n=20)$.

Based on PET imaging, patients were organized into three groups including (a) resectable CLM $(n=28)$, (b) unresectable CLM with "conversion" as a strategic treatment goal $(n=5)$, and (c) unresectable CLM with systematic therapy $(n=23)$.

PET imaging had consistent findings with conventional imaging in 37 patients, and PET had different findings in 19 patients. For 19 patients whose PET had different findings, PET downstaged in 4 patients and upstaged in 15 patients (Table 3). PET had a major impact in $10(17.9 \%)$ patients by modifying the therapy from unresectable to resectable in 4 patients, and modifying therapy from resectable to unresectable in 6 patients. PET had a minor impact in 4 (7.1\%) patients for changing the surgery extent. For the other 5 patients upstaging, there was no impact on their therapy plan. Above all, PET imaging had a clinical impact in $25 \%$ of patients. Each patient's condition and the clinical impact of PET are listed in Table 3. Major and minor impact for two typical cases are shown in Figures 6, 7, respectively.

\section{Patient Outcome}

Based on PET/CT and PET/MRI, patients were organized into three groups including (a) resectable CLM $(n=28)$, (b) unresectable CLM with "conversion" as a strategic treatment

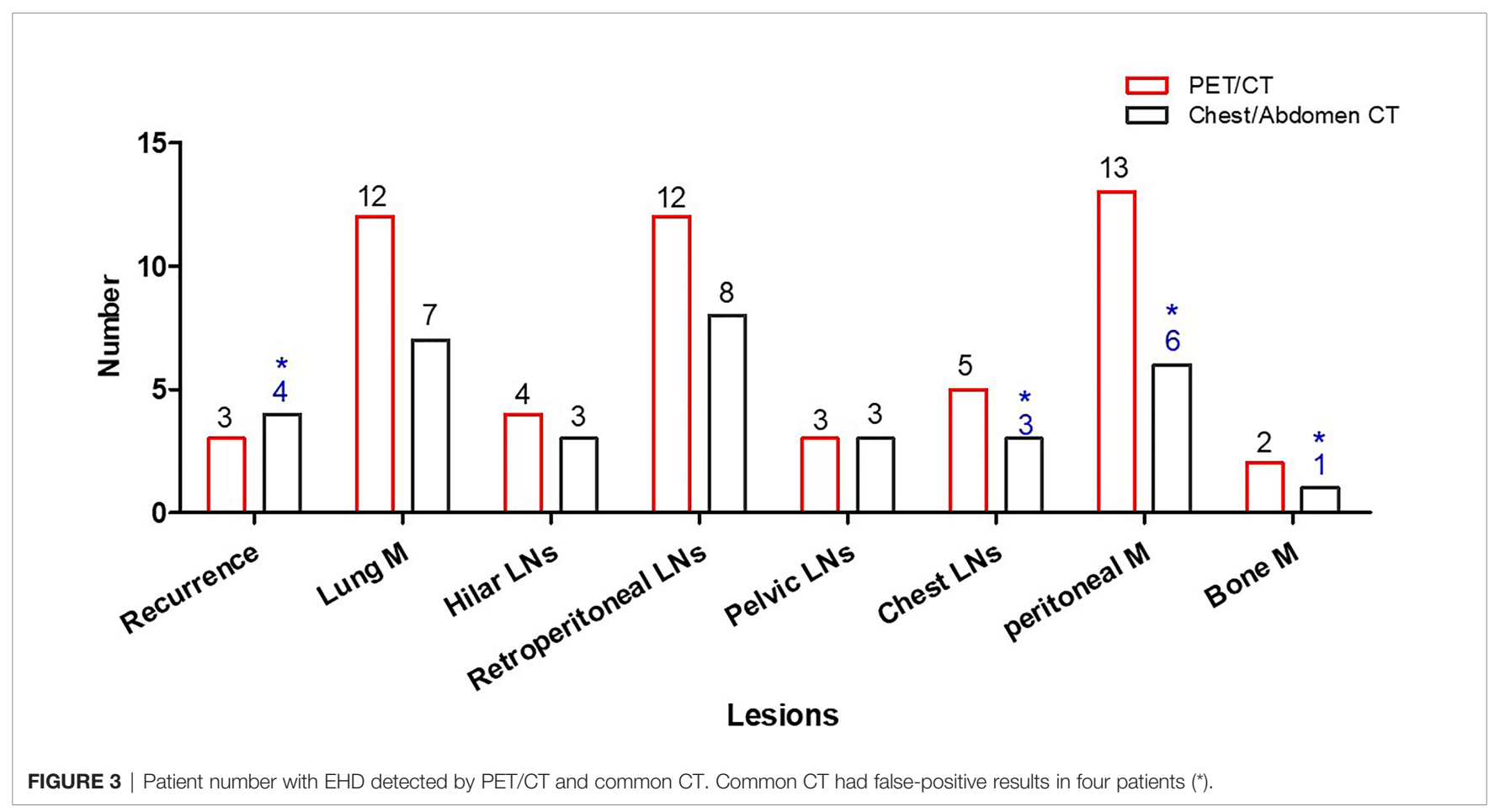




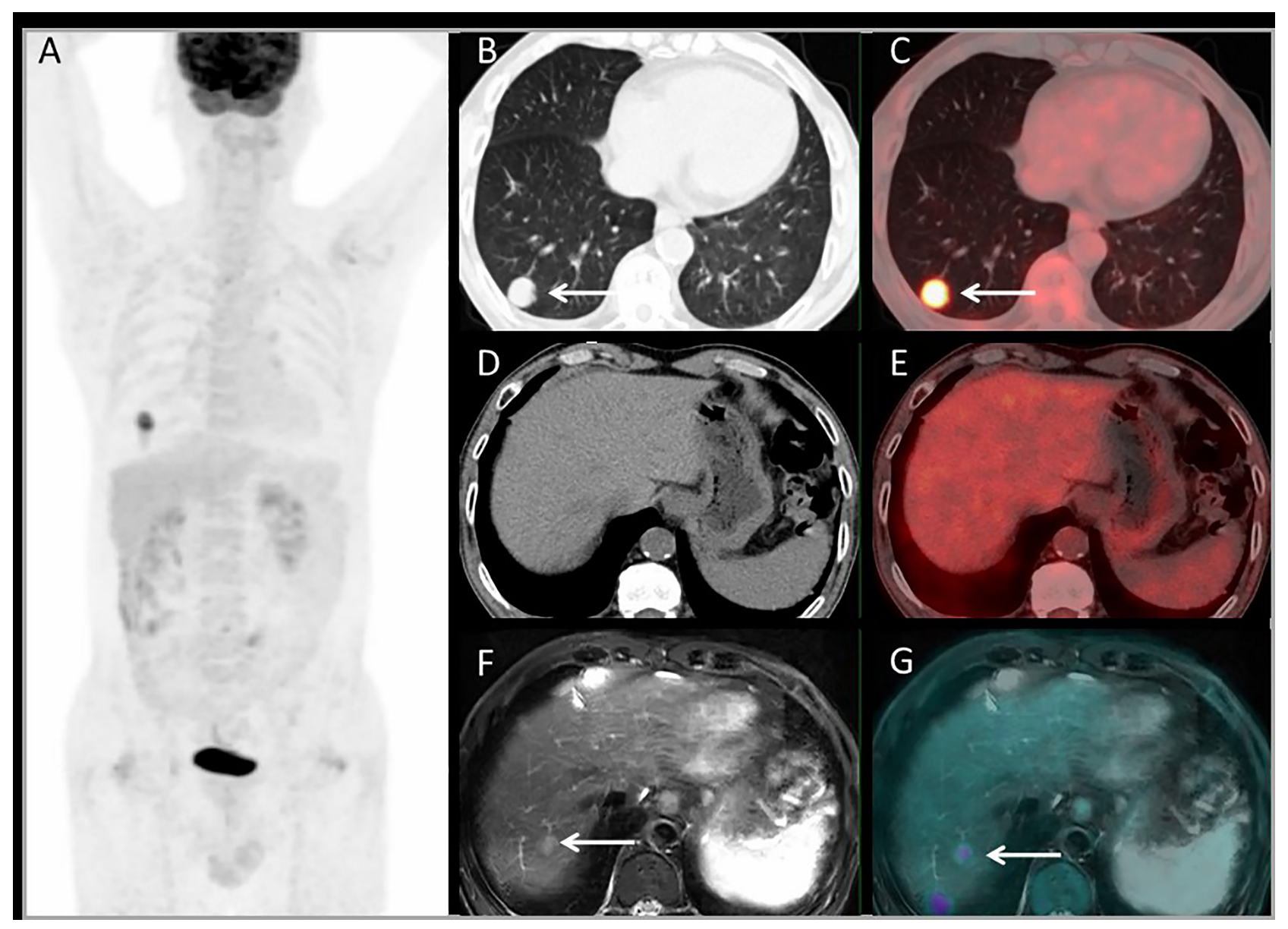

FIGURE 4 | Images of a 63-year-old male with colonic liver metastasis. The MIP (A) of PET/CT showed a lesion on right lung but no ${ }^{18} \mathrm{~F}-\mathrm{FDG}$ foci on liver. CT and $\mathrm{PET} / \mathrm{CT}$ fusion images showed single lung metastasis with high ${ }^{18} \mathrm{~F}-\mathrm{FDG}$ uptake (B, C) and no lesions were detected on liver (D, E). T2WI and PET/MRI fusion images showed a single lesion on right liver lobe with mild ${ }^{18} \mathrm{~F}$-FDG uptake $(\mathbf{F}, \mathbf{G})$.

goal $(n=5)$, and (c) unresectable CLM with systematic therapy $(n=23)$. Patient's outcome were as follows.

Group a: For 28 patients with resectable CLM, surgery was performed in 26 patients including liver $(n=10)$, combined liver and lung $(n=1)$, combined liver and upper abdominal nodules $(n=3)$, synchronous colon and liver resection $(n=$ $7)$, metachronous colon then liver resection $(n=5)$. And the other two patients received radiofrequency therapy $(n=2)$.

Group b: For five patients with "conversion" as a strategic treatment goal, all patients received systemic therapy. The resectability was evaluated 2 months later after optimal treatment and reevaluated 4 months later. At last, four patients received surgery and one patient received liver radiotherapy. For four patients who received surgery, three received liver lesion resection and one received liver lesion and abdominal nodules resection.

Group c: For 23 patients with unresectable CLM, 5 patients had non-operative liver lesion detected by PET/MRI, and 18 of these patients had non-operative EHD detected by PET/CT. They all received systematic therapy.

\section{DISCUSSION}

The current study investigated the role of PET/CT and abdomen PET/MRI in the preoperative work-up of patients with colorectal liver metastases under consideration for curative resection. The evidence from this study demonstrates that patients were upstaging from operative to non-operative disease $(10.7 \%)$, downstaging from non-operative to operative $(7.1 \%)$, and changing operation extent $(7.1 \%)$.

Reduced sensitivity in the detection of sub-centimeter lesions is an accepted limitation of PET/CT, while small liver metastases could be detected more reliably by CE-MRI or CE-CT than by PET/CT. In the authors' institution, it is a standard practice to use CE-MRI or CE-CT to depict the extent of hepatic disease and use conventional chest CT 


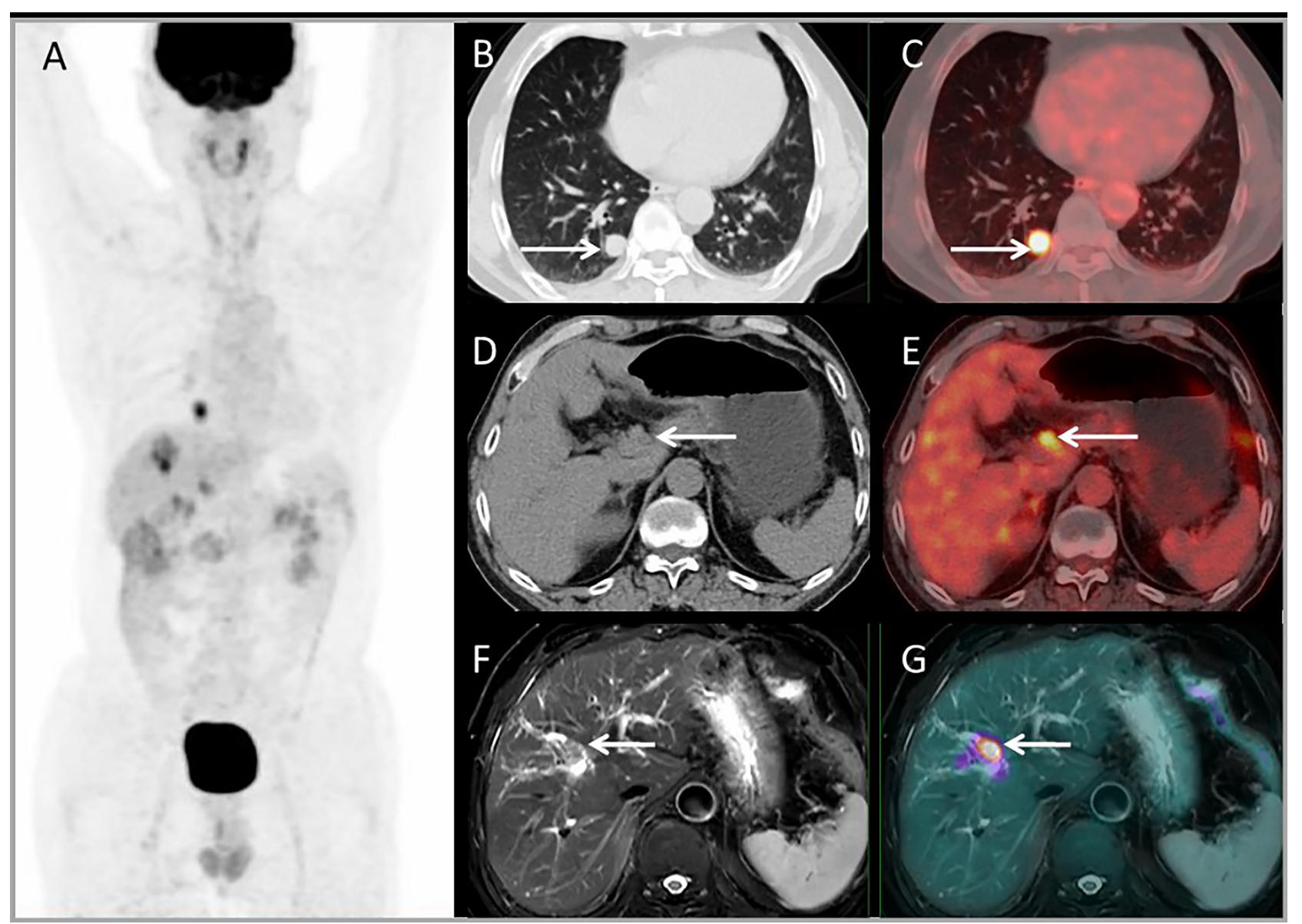

FIGURE 5 | Images of a 64-year-old male with colonic liver metastasis. The MIP (A) of PET/CT showed a single lesion on the liver and EHD lesions. CT and PET/CT fusion images showed single lung metastasis (B, C) and hilar lymph node metastasis (D, E). T2WI and PET/MR fusion images showed single liver lesion with intrahepatic bile duct infiltration (F, G).

and abdomen CT to stage extrahepatic disease prior to potential metastasis surgery; PET-CT is performed when conventional CT results are uncertain. In this study, PET-CT had detected more EHD than conventional imaging (60.7\% vs. $46.4 \%)$. A recently published meta-analysis study evaluating PET and PET/CT in patients with liver metastases reported that PET findings resulted in changes in the management of a mean of $24 \%$ of patients, with a mean incidence of PET-based EHD of $32 \%$ (22).

The current study shows that PET/MRI, as a new diagnostic modality, is feasible for accurate staging with regard to hepatic metastases. It provides a significantly higher diagnostic accuracy in the detection of liver metastases when compared to PET/CT (99.5\% vs. 47.5\%). Additionally, PET/MRI showed comparable ability with CE-CT (100\% vs. $86.5 \%)$ and CE-MRI (99.4\% vs. 99.4\%) for liver lesions. In an earlier trial, Brendle et al. reported that PET/MRI (MRI/DWI/PET) without contrast enhancement showed a relatively lower sensitivity $(71 \%)$, specificity $(80 \%)$, as well as diagnostic accuracy (74\%) for liver metastases in colorectal cancer. This was mainly because the data contained a relatively high percentage of mucinous tumors, which is known

TABLE 2 | Diagnostic accuracy of PET/CT and PET/MR compared with MRI/CT for CRLM.

\begin{tabular}{|c|c|c|c|}
\hline Group & CE-MRI & PET/MR & PET/CT \\
\hline Patient based $(n=43)$ & $43(100 \%)$ & $43(100 \%)$ & 32 (74.4\%) \\
\hline Lesion based $(n=169)$ & 168 (99.4\%) & 168 (99.4\%) & 82 (48.5\%) \\
\hline Group & CE-CT & PET/MR & PET/CT \\
\hline Patient based $(n=12)^{\star}$ & $13(100 \%)^{*}$ & $12(100 \%)$ & $6(58.3 \%)$ \\
\hline Lesion based $(n=37)$ & 32 (86.5\%) & $37(100 \%)$ & $16(43.2 \%)$ \\
\hline
\end{tabular}

${ }^{*}$ One patient with HCC misdiagnosed as metastasis by liver CE-CT while accurately diagnosed by PET/CT and PET/MR. 
TABLE 3 | Additional PET findings in all 19 patients and the clinical impact.

\begin{tabular}{|c|c|c|c|c|c|c|}
\hline Patients & $\begin{array}{l}\text { Convention Imaging } \\
\text { Finding }\end{array}$ & PET Imaging Finding & $\begin{array}{l}\text { Therapeutic } \\
\text { Comment by } \\
\text { Conventional } \\
\text { Imaging }\end{array}$ & $\begin{array}{c}\text { Therapy Comment by PET } \\
\text { Imaging }\end{array}$ & Stage & $\begin{array}{l}\text { Clinical Impact of } \\
\text { PET }\end{array}$ \\
\hline 1 & Recurrence & None & Unresectable & Resectable & Down & Major \\
\hline 2 & Mediastinal LN M & Inflammatory LN & Unresectable & Resectable & Down & Major \\
\hline 3 & $\begin{array}{l}\text { Suspicious right iliac } \\
\text { bone } \mathrm{M}\end{array}$ & None & Unresectable & Resectable & Down & Major \\
\hline 4 & Peritoneal nodule & None & Unresectable & Resectable & Down & Major \\
\hline 5 & Equivocal pelvic bone $\mathrm{M}$ & Bone/Peritoneal/retroperitoneal LNs M & Resectable & Unresectable & Up & Major \\
\hline 6 & None & $\begin{array}{l}\text { Bone/supraclavicular/mediastinal/ } \\
\text { retroperitoneal LNs M }\end{array}$ & Resectable & Unresectable & Up & Major \\
\hline 7 & None & Recurrent/Peritoneal Nodule M & Resectable & Unresectable & Up & Major \\
\hline 8 & None & Peritoneal Nodule M & Resectable & Unresectable & Up & Major \\
\hline 9 & None & Retroperitoneal LN & Resectable & Unresectable & Up & Major \\
\hline 10 & None & Peritoneal Nodule M & Resectable & Unresectable & Up & Major \\
\hline 11 & $\begin{array}{l}\text { Equivocal small lung } \\
\text { nodule }\end{array}$ & Lung M/Hilar LN & Resectable & Resectable & Up & Minor \\
\hline 12 & No & Hilar LN & Resectable & Resectable & Up & Minor \\
\hline 13 & Retroperitoneal LN & Hilar LN/Retroperitoneal LN & Resectable & Resectable & Up & Minor \\
\hline 14 & $\begin{array}{l}\text { Abdomen Peritoneal } \\
\text { Nodule }\end{array}$ & Abdomen/Pelvic Peritoneal Nodule & Resectable & Resectable & Up & Minor \\
\hline 15 & $\begin{array}{l}\text { Equivocal small lung } \\
\text { nodule }\end{array}$ & Lung $\mathrm{M}$ & Resectable & Resectable & Up & No \\
\hline 16 & $\begin{array}{l}\text { Equivocal small lung } \\
\text { nodule }\end{array}$ & Lung $\mathrm{M}$ & Resectable & Resectable & Up & No \\
\hline 17 & $\begin{array}{l}\text { Lung inflammatory } \\
\text { nodule }\end{array}$ & Lung $\mathrm{M}$ & Resectable & Resectable & Up & No \\
\hline 18 & Lung M/Mediastinal LN & Lung M/Mediastinal LN/Recurrent & Unresectable & Unresectable & Up & No \\
\hline 19 & Retroperitoneal LN & Retroperitoneal LN/Peritoneal Nodule & Unresectable & Unresectable & Up & No \\
\hline
\end{tabular}

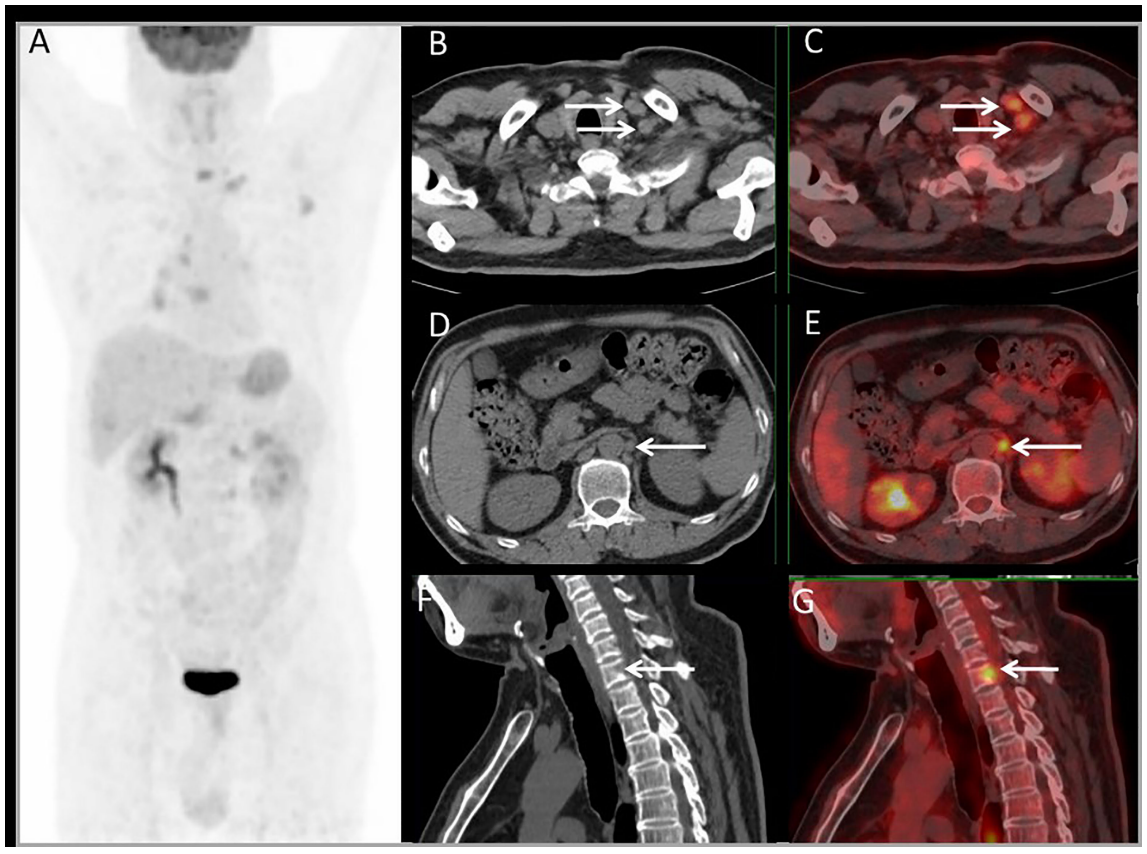

FIGURE 6 | Images of a 54-year-old male with rectal liver metastases. Common CT showed no EHD. The MIP (A) of PET/CT showed liver metastasis and multiple EHD. PET and PET/CT fusion images showed left supraclavicular lymph nodes metastases (B, C), retroperitoneal lymph nodes metastases (D, E), and bone metastases on the 7 th cervical vertebra $\mathbf{( F , ~ G ) . ~ T h e ~ t h e r a p y ~ s t r a t e g y ~ w a s ~ c h a n g e d ~ f r o m ~ r e s e c t a b l e ~ t o ~ u n r e s e c t a b l e ~ ( m a j o r ~ i m p a c t ) . ~ T h e ~ p a t i e n t ~ r e c e i v e d ~ s y s t e m a t i c ~}$ therapy after PET/CT restaging. 


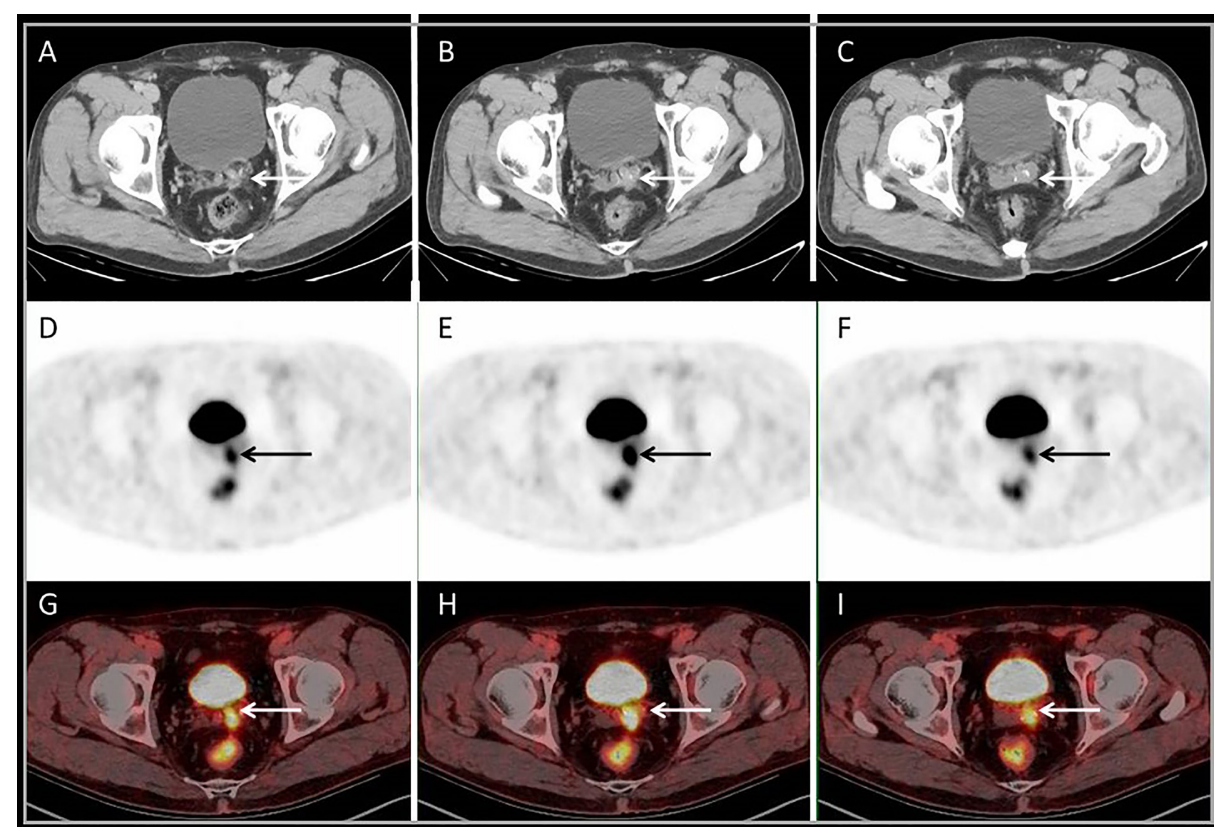

FIGURE 7 | Images of a 52-year-old male with single liver metastasis after surgery of sigmoid cancer resection. Conventional abdomen CT showed no EHD (A-C). PET and PET/CT fusion images showed a peritoneal nodule adjacent to the left seminal vesicle gland (D-I). The surgery extent changed from liver only to combining live and peritoneal nodule (minor impact).

to be challenging for either DWI or PET evaluation (28). Hybrid PET/MRI with contrast enhancement showed higher accuracy for liver metastases (sensitivity 92\%-100\%, specificity $97 \%-$ $100 \%)$ (29-31). This was consistent with the current findings despite the different acquisition procedures, as this study did not use contrast agents.

There are some limitations in this study. The patients were superselected with CLM, which is diagnosed by CE-CT or CEMRI ahead. Therefore, radiologists were not blinded to the patients' history. Considering the expenditure of time and a questionable added value of PET/MRI, we did not use liverspecific contrast agent. Histopathological confirmation of every detected lesion was not available due to ethical and practical reasons. In addition, the cost-effectiveness of this imaging modality was not evaluated. We consider that the additional cost of PET-CT can be largely offset by the reduction in number of futile invasive or operative procedures, which certainly need further investigation.

\section{CONCLUSION}

The results of this study indicate that PET/CT is valuable in EHD detection. Abdomen PET/MRI showed comparable ability with CE-CT/CE-MRI in liver lesion detection. PET imaging had a clinical impact on $25 \%$ of patients. Hence, a simultaneous wholebody PET/CT and abdomen PET/MRI may become a new onestop imaging method in the preoperative work-up of patients with potentially resectable colorectal liver metastases.

\section{DATA AVAILABILITY STATEMENT}

The original contributions presented in the study are included in the article/Supplementary Material. Further inquiries can be directed to the corresponding authors.

\section{ETHICS STATEMENT}

The studies involving human participants were reviewed and approved by the Medical Ethics Committee of Peking University Cancer Hospital (ethical approval no. 2018KT110-GZ01). The patients/participants provided their written informed consent to participate in this study. Written informed consent was obtained from the individual(s) for the publication of any potentially identifiable images or data included in this article.

\section{AUTHOR CONTRIBUTIONS}

All authors listed have made a substantial, direct, and intellectual contribution to the work and approved it for publication.

\section{FUNDING}

The current research was financially supported by the National Natural Science Foundation (No. 81871387), Beijing Natural Science Foundation, Jing-Jin-Ji special projects for basic 
research cooperation (H2018206600), Beijing Excellent Talents Funding (2017000021223ZK33), Beijing Municipal Administration of Hospitals-Yangfan Project (ZYLX201816), and Science Foundation of Peking Univesity Cancer Hospital (No. 2021-4).

\section{REFERENCES}

1. Scheele J, Stang R, Altendorfhofmann A, Paul M. Resection of Colorectal Liver Metastases. World J Surg (1995) 19:59-71. doi: 10.1007/BF00316981

2. Rees M, Tekkis PP, Welsh FKS, O’Rourke T, John TG. Evaluation of LongTerm Survival After Hepatic Resection for Metastatic Colorectal Cancer - A Multifactorial Model of 929 Patients. Ann Surg (2008) 247:125-35. doi: 10.1097/SLA.0b013e31815aa2c2

3. Taylor A, Kanas G, Primrose J, Langeberg W, Alexander D, Kelsh M, et al. Survival After Surgical Resection of Hepatic Metastases From Colorectal Cancer: An Updated Review and Meta-Analysis. Ann Oncol (2011) 22:v21-1.

4. Simmonds PC, Primrose JN, Colquitt JL, Garden OJ, Poston GJ, Rees M. Surgical Resection of Hepatic Metastases From Colorectal Cancer: A Systematic Review of Published Studies. Br J Cancer (2006) 94:982-99. doi: $10.1038 /$ sj.bjc.6603033

5. Carpizo DR, D’Angelica M. Liver Resection for Metastatic Colorectal Cancer in the Presence of Extrahepatic Disease. Lancet Oncol (2009) 10:801-9. doi: 10.1016/S1470-2045(09)70081-6

6. Pawlik TM, Schulick RD, Choti MA. Expanding Criteria for Resectability of Colorectal Liver Metastases. Oncologist (2008) 13:51-64. doi: 10.1634/ theoncologist.2007-0142

7. Meng XP, Wang YC, Ju S, Lu CQ, Zhong BY, Ni CF, et al. Radiomics Analysis on Multiphase Contrast-Enhanced CT: A Survival Prediction Tool in Patients With Hepatocellular Carcinoma Undergoing Transarterial Chemoembolization. Front Oncol (2020) 10:1196. doi: 10.3389/ fonc. 2020.01196

8. Li B, Feng F, Jia H, Jiang Q, Cao S, Wei L, et al. Rhamnetin Decelerates the Elimination and Enhances the Antitumor Effect of the Molecular-Targeting Agent Sorafenib in Hepatocellular Carcinoma Cells via the miR-148a/PXR Axis. Food Funct (2021) 12:2404-17. doi: 10.1039/D0FO02270E

9. Floriani I, Torri V, Rulli E, Garavaglia D, Compagnoni A, Salvolini L, et al. Performance of Imaging Modalities in Diagnosis of Liver Metastases From Colorectal Cancer: A Systematic Review and Meta-Analysis. J Magn Reson Imaging (2010) 31:19-31. doi: 10.1002/jmri.22010

10. Niekel MC, Bipat S, Stoker J. Diagnostic Imaging of Colorectal Liver Metastases With CT, MR Imaging, FDG PET, and/or FDG PET/CT: A Meta-Analysis of Prospective Studies Including Patients Who Have Not Previously Undergone Treatment. Radiology (2010) 257:674-84. doi: 10.1148/radiol.10100729

11. He M, Zhang P, Ma X, He B, Fang C, Jia F. Radiomic Feature-Based Predictive Model for Microvascular Invasion in Patients With Hepatocellular Carcinoma. Front Oncol (2020) 10:574228. doi: 10.3389/fonc.2020.574228

12. Zech CJ, Korpraphong P, Huppertz A, Denecke T, Kim MJ, Tanomkiat W, et al. Randomized Multicentre Trial of Gadoxetic Acid-Enhanced MRI Versus Conventional MRI or CT in the Staging of Colorectal Cancer Liver Metastases (Vol 101, pg 613, 2014). Br J Surg (2014) 101:613-21. doi: 10.1002/bjs.9465

13. Zhang L, Kuang S, Chen J, Zhang Y, Zhao B, Peng H, et al. The Role of Preoperative Dynamic Contrast-Enhanced 3.0-T MR Imaging in Predicting Early Recurrence in Patients With Early-Stage Hepatocellular Carcinomas After Curative Resection. Front Oncol (2019) 9:1336. doi: 10.3389/ fonc. 2019.01336

14. Sung PS, Choi MH, Yang H, Lee SK, Chun HJ, Jang JW, et al. DiffusionWeighted Magnetic Resonance Imaging in Hepatocellular Carcinoma as a Predictor of a Response to Cisplatin-Based Hepatic Arterial Infusion Chemotherapy. Front Oncol (2020) 10:600233. doi: 10.3389/fonc.2020.600233

15. Zhou W, Jian W, Cen X, Zhang L, Guo H, Liu Z, et al. Prediction of Microvascular Invasion of Hepatocellular Carcinoma Based on ContrastEnhanced MR and 3D Convolutional Neural Networks. Front Oncol (2021) 11:588010. doi: 10.3389/fonc.2021.588010

\section{SUPPLEMENTARY MATERIAL}

The Supplementary Material for this article can be found online at: https://www.frontiersin.org/articles/10.3389/fonc.2021. 714948/full\#supplementary-material

16. Mainenti PP, Mancini M, Mainolfi C, Camera L, Maurea S, Manchia A, et al. Detection of Colo-Rectal Liver Metastases: Prospective Comparison of Contrast Enhanced US, Multidetector CT, PET/CT, and 1.5 Tesla MR with extracellular and reticulo-endothelial cell specific contrast agents. Abdom Imaging (2010) 35:511-21. doi: 10.1007/s00261-009-9555-2

17. Xu H, Guo W, Cui X, Zhuo H, Xiao Y, Ou X, et al. Three-Dimensional Texture Analysis Based on PET/CT Images to Distinguish Hepatocellular Carcinoma and Hepatic Lymphoma. Front Oncol (2019) 9:844. doi: 10.3389/ fonc.2019.00844

18. Li R, Li D, Jia G, Li X, Sun G, Zuo C. Diagnostic Performance of Theranostic Radionuclides Used in Transarterial Radioembolization for Liver Cancer. Front Oncol (2020) 10:551622. doi: 10.3389/fonc.2020.551622

19. Hendlisz A, Golfinopoulos V, Garcia C, Covas A, Emonts P, Ameye L, et al. Serial FDG-PET/CT for Early Outcome Prediction in Patients With Metastatic Colorectal Cancer Undergoing Chemotherapy. Ann Oncol (2012) 23:1687-93. doi: 10.1093/annonc/mdr554

20. Moulton C, Levine MN, Law C, Hart R, Ruo L, Gu C, et al. An Ontario Clinical Oncology Group (OCOG) Randomized Controlled Trial (RCT) Assessing FDG PET/CT in Resectable Liver Colorectal Adenocarcinoma Metastases (CAM). J Clin Oncol (2011) 29(4):3520. doi: 10.1200/jco.2011.29.15_suppl.3520

21. Wang T, Zhang KH. New Blood Biomarkers for the Diagnosis of AFPNegative Hepatocellular Carcinoma. Front Oncol (2020) 10:1316. doi: $10.3389 /$ fonc. 2020.01316

22. Maffione AM, Lopci E, Bluemel C, Giammarile F, Herrmann K, Rubello D. Diagnostic Accuracy and Impact on Management of F-18-FDG PET and PET/CT in Colorectal Liver Metastasis: A Meta-Analysis and Systematic Review. Eur J Nucl Med Mol Imaging (2015) 42:152-63. doi: 10.1007/ s00259-014-2930-4

23. Delso G, Furst S, Jakoby B, Ladebeck R, Ganter C, Nekolla SG, et al. Performance Measurements of the Siemens mMR Integrated Whole-Body PET/MR Scanner. J Nucl Med (2011) 52:1914-22. doi: 10.2967/ jnumed.111.092726

24. Martin O, Schaarschmidt BM, Kirchner J, Suntharalingam S, Grueneisen J, Demircioglu A, et al. PET/MRI Versus PET/CT in Whole-Body Staging: Results From a Unicenter Observational Study in 1003 Subsequent Examinations. J Nucl Med (2020) 61(8):1131-6. doi: 10.2967/ jnumed.119.233940

25. Mayerhoefer ME, Prosch H, Beer L, Tamandl D, Beyer T, Hoeller C, et al. PET/MRI Versus PET/CT in Oncology: A Prospective Single-Center Study of 330 Examinations Focusing on Implications for Patient Management and Cost Considerations. Eur J Nucl Med Mol Imaging (2020) 47:51-60. doi: 10.1007/s00259-019-04452-y

26. Sörensen J, Velikyan I, Sandberg D, Wennborg A, Feldwisch J, Tolmachev V, et al. Measuring HER2-Receptor Expression In Metastatic Breast Cancer Using [68ga]ABY-025 Affibody PET/Ct. Theranostics (2016) 6:262-71. doi: 10.7150/thno.13502

27. Padhani AR, Koh DM, Collins DJ. Whole-Body Diffusion-Weighted MR Imaging in Cancer: Current Status and Research Directions. Radiology (2011) 261:700-18. doi: 10.1148/radiol.11110474

28. Brendle C, Schwenzer NF, Rempp H, Schmidt H, Pfannenberg C, la Fougere C, et al. Assessment of Metastatic Colorectal Cancer With Hybrid Imaging: Comparison of Reading Performance Using Different Combinations of Anatomical and Functional Imaging Techniques in PET/MRI and PET/CT in a Short Case Series. Eur J Nucl Med Mol Imaging (2016) 43:123-32. doi: 10.1007/s00259-015-3137-z

29. Kirchner J, Sawicki LM, Deuschl C, Gruneisen J, Beiderwellen K, Lauenstein TC, et al. 18 F-FDG PET/MR Imaging in Patients With Suspected Liver Lesions: Value of Liver-Specific Contrast Agent Gadobenate Dimeglumine. PloS One (2017) 12:e0180349. doi: 10.1371/journal.pone.0180349 
30. Beiderwellen K, Geraldo L, Ruhlmann V, Heusch P, Gomez B, Nensa F, et al. Accuracy of [18F]FDG PET/MRI for the Detection of Liver Metastases. PloS One (2015) 10:e0137285. doi: 10.1371/journal.pone.0137285

31. Melsaether AN, Raad RA, Pujara AC, Ponzo FD, Pysarenko KM, Jhaveri K, et al. Comparison of Whole-Body (18)F FDG PET/MR Imaging and WholeBody (18)F FDG PET/CT in Terms of Lesion Detection and Radiation Dose in Patients With Breast Cancer. Radiology (2016) 281:193-202. doi: 10.1148/ radiol.2016151155

Conflict of Interest: The authors declare that the research was conducted in the absence of any commercial or financial relationships that could be construed as a potential conflict of interest.
Publisher's Note: All claims expressed in this article are solely those of the authors and do not necessarily represent those of their affiliated organizations, or those of the publisher, the editors and the reviewers. Any product that may be evaluated in this article, or claim that may be made by its manufacturer, is not guaranteed or endorsed by the publisher.

Copyright $\odot 2021$ Zhou, Guo, Sun, Yu, Zhu, Li and Yang. This is an open-access article distributed under the terms of the Creative Commons Attribution License (CC BY). The use, distribution or reproduction in other forums is permitted, provided the original author(s) and the copyright owner(s) are credited and that the original publication in this journal is cited, in accordance with accepted academic practice. No use, distribution or reproduction is permitted which does not comply with these terms. 